\title{
High Order Three-Steps Newton Raphson-like Schemes for Solving Nonlinear Equation Systems
}

\author{
Rizki Multazamil Fatahillah, M Ziaul Arif*, Rusli Hidayat, Kusbudiono, Ikhsanul \\ Halikin
}

Department of Mathematics, FMIPA, University of Jember

${ }^{*}$ Corresponding author. Email: ziaul.fmipa@unej.ac.id

\begin{abstract}
This study proposes several new 3-steps schemes based on the Newton-Raphson method for solving non-linear equation systems. The proposed schemes are analysed and formulated based on the Newton-Raphson method and the Newtoncotes open form numerical integration method. In general, the schemes can be considered as a predictor and corrector principles. In the first and the third steps, the Newton-Raphson method is applied. Furthermore, Newton-cotes Open Form numerical integration modification is operated in the second step of the proposed schemes. The convergence analysis of the proposed schemes is given. It shows that the proposed scheme provides the $8^{\text {th }}$ order of convergence. The performance of the proposed schemes is compared and assessed with several numerical examples.
\end{abstract}

Keywords: Nonlinear equation systems, Newton-Raphson method, Newton-cotes open form.

\section{INTRODUCTION}

Finding the exact or nearly exact solution of the nonlinear equation system is the most common problem in mathematics. Since the exact solution, called the analytical solution is problematic in some mathematical sense, the numerical solution provides the solution that produces a nearly exact solution of the problems. The algorithm is considered to be effective and efficient if it has a high order of convergence. It means the algorithm can produce the solution faster. Several modifications of algorithms have been introduced and investigated [1-7]. However, discovering the other high order algorithm is highly possible.

Some modifications and new schemes for solving have been investigated for decades. Farida in [1] proposed some 3-steps Newton method schemes by utilizing the numerical integration to modify the steps of the schemes. The schemes have an order of convergence 6. Furthermore, Frontini and Sormani [2], denoted FSM, found three-steps $5^{\text {th }}$ and $6^{\text {th }}$-order of convergence scheme based on predictor-corrector principle. Moreover, Darvishi et al. [3-4] investigated and proposed two new methods: Newton-like $3^{\text {rd }}$-order of convergence method and super cubic iterative approach to solve nonlinear equation system. Khirallah and Hafiz developed $3^{\text {rd }}$-order Newton-family and Jarrat methods to solve non- linear equation systems in 2012 [7], called KHM methods.

There are still many possibilities to develop and modify non-linear equation system solving methods to get a higher-order convergence. The modification method can be conducted by using either one-step or multi-step methods. In this paper, the first and third steps are the Newton-Raphson method. Furthermore, the modification of the 3-steps method has been done by employing numerical integration Newton-cotes Open Form six and seven points method as the second step of the proposed method.

\section{METHODS}

\subsection{System of Nonlinear Equations (SNLE)}

The general form of non-linear equation system is

$\mathbf{F}\left(x_{1}, x_{2}, \ldots, x_{m}\right)=$

$\left(f_{1}\left(x_{1}, x_{2}, \ldots, x_{m}\right), f_{2}\left(x_{1}, x_{2}, \ldots, x_{m}\right), \ldots, f_{m}\left(x_{1}, x_{2}, \ldots, x_{m}\right)\right)$.

Where $f_{i}$ is the non-linear function which is mapping $\mathbb{R}^{m}$ into $\mathbb{R}^{m}$. The system of the function $F$ is mapping $\mathbb{R}^{m}$ into $\mathbb{R}^{m}$. Furthermore, the solution of the system is $x^{*}$, if $F\left(x^{*}\right)=0$.

The solution of the non-linear equation systems can be obtained by using iterative methods. The most 
common iterative method is the Newton-Raphson method. However, the modifications of the NewtonRaphson method have been investigated in some references.

\subsection{Numerical Integration}

In this paper, the numerical integration method is used for modifying the non-linear equation system schemes. High order numerical integration Newton-Cotes open forms is employed as the second step of the proposed method. The numerical integrations Newton-Cotes open forms 4, 5, 6 and 7-points are modified in this paper.

\section{RESULTS}

\subsection{Modification of the Schemes}

Let $X$ be the solution of the differentiated function and considered as the numerical solution of the equation system $F(x)=0$, for $F: D \subseteq \mathbb{R}^{n} \rightarrow \mathbb{R}^{n}$ is the mapping that continues in the set $D$ convex, $F(x)$ has unique roots in $D,(x)=\left(f_{1}(x), f_{2}(x), \ldots, f_{n}(x)\right) T$

$x=\left(x_{1}, x_{2}, \ldots, x_{n}\right)^{T}$ and $f_{i}: \mathbb{R}^{n} \rightarrow \mathbb{R}$ is a nonlinear function, then:

$$
F(x)=F\left(x_{i}\right)+\int_{\mathrm{xi}}^{\mathrm{x}} F^{\prime}(t) d t .
$$

If the integral of the Equation (1) is approximated with numerical integration method, Newton-Cotes open form 6-points, it can be written as

$$
\begin{aligned}
\int_{x_{0}}^{x_{7}} F^{\prime}(x) d x=\frac{7 h}{1440}[6 & 611 F^{\prime}\left(\frac{6 x_{0}+x_{7}}{7}\right)-453 F^{\prime}\left(\frac{5 x_{0}+2 x_{7}}{7}\right) \\
+ & 562 F^{\prime}\left(\frac{4 x_{0}+3 x_{7}}{7}\right)+562 F^{\prime}\left(\frac{3 x_{0}+4 x_{7}}{7}\right) \\
- & \left.453 F^{\prime}\left(\frac{2 x_{0}+5 x_{7}}{7}\right)+611 F^{\prime}\left(\frac{x_{0}+6 x_{7}}{7}\right)\right] .
\end{aligned}
$$

Supposed that $x_{0}=x_{i}$ and $x_{7}=x, h=\frac{x-x_{i}}{7}$, and supposed that $F(x)$ is a system of nonlinear equations. It can be assumed that a vector $\left(x^{*}\right)$ is a solution of $F(x)$, such that $F\left(x^{*}\right)=0$. By substituting Equation (2) to Equation (1), the implicit scheme of solving the nonlinear equation systems can be formed,

$$
\begin{aligned}
x=x_{i}-1440\left[611 F^{\prime}\right. & \left(\frac{6 x_{i}+x}{7}\right)-453 F^{\prime}\left(\frac{5 x_{i}+2 x}{7}\right) \\
+ & 562 F^{\prime}\left(\frac{4 x_{i}+3 x}{7}\right) \\
+ & 562 F^{\prime}\left(\frac{3 x_{i}+4 x}{7}\right) \\
& -453 F^{\prime}\left(\frac{2 x_{i}+5 x}{7}\right) \\
+ & \left.611 F^{\prime}\left(\frac{x_{i}+6 x}{7}\right)\right]^{-1} F\left(x_{i}\right)
\end{aligned}
$$

The order of convergence of Equation (3) can be increased by modifying the interpolation points using the formula $\frac{(N-M) x_{i}+M x_{i}}{N}$ where $\mathrm{N}$ and $\mathrm{M}$ are integers, see [1] for the details of the interpolation points. Once the linear equation of the interpolation points has been determined, the non-unique solution of the linear equation can be found. Consequently, the new interpolation points of Equation (3) are such equations below,

$w_{1}=\frac{3 x_{i}+4 y_{i}}{7}, w_{2}=\frac{-2 x_{i}+9 y_{i}}{7}, w_{3}=\frac{-x_{i}+8 y_{i}}{7}, w_{4}=\frac{x_{i}+6 y_{i}}{7}$., $w_{5}=\frac{2 x_{i}+5 y_{i}}{7}$, dan $w_{6}=\frac{-3 x_{i}+10 y_{i}}{7}$

Finally, Equation (3) can be written using the new interpolation points as follows,

$$
\begin{aligned}
x=x_{i}-1440\left[611 F^{\prime}\right. & \left(\frac{3 x_{i}+4 x}{7}\right)-453 F^{\prime}\left(\frac{-2 x_{i}+9 x}{7}\right) \\
& +562 F^{\prime}\left(\frac{-x_{i}+8 x}{7}\right) \\
& +562 F^{\prime}\left(\frac{x_{i}+6 x}{7}\right) \\
& -453 F^{\prime}\left(\frac{2 x_{i}+5 x}{7}\right) \\
+ & \left.611 F^{\prime}\left(\frac{-3 x_{i}+10 x}{7}\right)\right]^{-1} F\left(x_{i}\right)
\end{aligned}
$$

Equation (4) is an implicit equation. However, since the converged solution is expected in this case, we can assume the Equation (4) is an explicit equation if the value of $x$ on the right-hand side is estimated with the prediction step from the Newton-Raphson scheme. Furthermore, by adding one step in the following step 2 (Equation (4), the new 3-steps Newton-Raphson-like scheme can be considered as the predictor-corrector technique as follows

$$
\begin{aligned}
& y_{n}=x_{n}-F^{\prime}\left(x_{n}\right)^{-1} F\left(x_{n}\right), \\
& Z_{n}=y_{n}-1440\left[611 F^{\prime}\left(\frac{3 x_{n}+4 y_{n}}{7}\right)-\right. \\
& 453 F^{\prime}\left(\frac{-2 x_{n}+9 y_{n}}{7}\right)+ \\
& 562 F^{\prime}\left(\frac{-x_{n}+8 y_{n}}{7}\right)+562 F^{\prime}\left(\frac{x_{n}+6 y_{n}}{7}\right)- \\
& 453 F^{\prime}\left(\frac{2 x_{n}+5 y_{n}}{7}\right)+ \\
&\left.611 F^{\prime}\left(\frac{-3 x_{n}+10 y_{n}}{7}\right)\right]^{-1} F\left(y_{n}\right), \\
& x_{n+1}= Z_{n}-F^{\prime}\left(Z_{n}\right)^{-1} F\left(Z_{n}\right) .
\end{aligned}
$$

Moreover, let the integral of the Equation (1) be approximated with numerical integration Newton-Cotes open form 7-points method. To increase the order of the convergence, we apply a similar manner to find the new interpolation points. Furthermore, the new 3-steps Newton-Raphson-like scheme can be determined as follows,

$$
\begin{aligned}
y_{n}=x_{n}-F^{\prime}\left(x_{n}\right)^{-1} F & \left(x_{n}\right) \\
Z_{n}=y_{n}-945\left[460 F^{\prime}\right. & \left(\frac{16 x_{n}-8 y_{n}}{8}\right)-954 F^{\prime}\left(\frac{-3 x_{n}+11 y_{n}}{8}\right) \\
& +2196 F^{\prime}\left(\frac{25 x_{n}-17 y_{n}}{8}\right) \\
& -2496 F^{\prime}\left(\frac{23 x_{n}-15 y_{n}}{8}\right) \\
+ & 2196 F^{\prime}\left(\frac{-2 x_{n}+10 y_{n}}{8}\right) \\
& -954 F^{\prime}\left(\frac{3 x_{n}+5 y_{n}}{8}\right) \\
& \left.+460 F^{\prime}\left(\frac{-x_{n}+9 y_{n}}{8}\right)\right]^{-1} F\left(y_{n}\right)
\end{aligned}
$$




$$
x_{n+1}=Z_{n}-F^{\prime}\left(Z_{n}\right)^{-1} F\left(Z_{n}\right)
$$

By using numerical integration method NewtonCotes open form 4-points to estimate the integration part of Equation (1), the following third 3-steps NewtonRaphson-like has been developed.

$$
\begin{gathered}
y_{n}=x_{n}-F^{\prime}\left(x_{n}\right)^{-1} F\left(x_{n}\right) \\
\begin{aligned}
Z_{n}=y_{n}-24\left[11 F^{\prime}\right. & \left(\frac{7 x_{n}-y_{n}}{6}\right)+F^{\prime}\left(\frac{2 x_{n}+4 y_{n}}{6}\right) \\
& +F^{\prime}\left(\frac{-2 x_{n}+8 y_{n}}{6}\right) \\
& \left.+11 F^{\prime}\left(\frac{-7 x_{n}+13 y_{n}}{6}\right)\right]^{-1} F\left(y_{n}\right)
\end{aligned} \\
x_{n+1}=Z_{n}-F^{\prime}\left(Z_{n}\right)^{-1} F\left(Z_{n}\right)
\end{gathered}
$$

Lastly, the integral of the Equation (1) can be approximated with numerical integration method Newton-Cotes open form 5-points. The modification of the numerical integration equation above yields the fourth 3-steps Newton-Raphson-like scheme below,

$$
\begin{aligned}
& y_{n}=x_{n}-F^{\prime}\left(x_{n}\right)^{-1} F\left(x_{n}\right) \\
& \begin{array}{r}
z_{n}=y_{n}-20\left[11 F^{\prime}\left(\frac{28 x_{n}-20 y_{n}}{8}\right)-14 F^{\prime}\left(\frac{11 x_{n}-3 y_{n}}{8}\right)\right. \\
\quad+26 F^{\prime}\left(\frac{-5 x_{n}+13 y_{n}}{8}\right)-14 F^{\prime}\left(\frac{19 x_{n}-11 y_{n}}{8}\right) \\
\left.+11 F^{\prime}\left(\frac{22 x_{n}-14 y_{n}}{8}\right)\right]^{-1} F\left(y_{n}\right)
\end{array} \\
& x_{n+1}=Z_{n}-F^{\prime}\left(Z_{n}\right)^{-1} F\left(Z_{n}\right)
\end{aligned}
$$

Four new 3-steps schemes have been established. Furthermore, the convergence analysis of all proposed schemes is explained in Section 3.2.

\subsection{Convergence Analysis}

\section{Algorithm \#1}

Theorem 1: Let $x^{*}$ be a simple solution of the differentiable function $F(x)$ and given the initial value $x_{i}$, the three-steps scheme defined in Equation (5) has order of convergence 8 .

\section{Proof.}

Step 1:

$$
y_{n}=x_{n}-\frac{F\left(x_{n}\right)}{F^{\prime}\left(x_{n}\right)} .
$$

By using the Taylor series, it can be obtained,

$$
\begin{aligned}
& F\left(x_{n}\right)=F\left(x^{*}\right)+F^{\prime}\left(x^{*}\right)\left(x_{n}-x^{*}\right)+F^{\prime \prime}\left(x^{*}\right) \frac{\left(x_{n}-x^{*}\right)^{2}}{2 !}+ \\
& F^{\prime \prime \prime}\left(x^{*}\right) \frac{\left(x_{n}-x^{*}\right)^{3}}{3 !}+\cdots
\end{aligned}
$$

Since $F\left(x^{*}\right)=0$, by assuming $V_{k}=\frac{1}{k !} \cdot \frac{F^{(k)}\left(x^{*}\right)}{F^{\prime}\left(x^{*}\right)}, k=$ $2,3, \ldots$ and $E_{n}=x_{n}-x^{*}$, then Equation (10) become,

$$
\begin{gathered}
F\left(x_{n}\right)=F^{\prime}\left(x^{*}\right)\left[E_{n}+V_{2} E_{n}{ }^{2}+V_{3} E_{n}{ }^{3}+V_{4} E_{n}{ }^{4}\right. \\
+V_{5} E_{n}{ }^{5}+V_{6} E_{n}{ }^{6}+V_{7} E_{n}{ }^{7} \\
\left.+V_{8} E_{n}{ }^{8}+O\left(\left\|E_{n}{ }^{9}\right\|\right)\right] .
\end{gathered}
$$

$F^{\prime}\left(x_{n}\right)=F^{\prime}\left(x^{*}\right)\left[I+2 V_{2} E_{n}+3 V_{3} E_{n}{ }^{2}+4 V_{4} E_{n}{ }^{3}+\right.$

$\left.V_{5} E_{n}{ }^{4}+6 V_{6} E_{n}{ }^{5}+7 V_{7} E_{n}{ }^{6}+8 V_{8} E_{n}{ }^{7}+O\left(\left\|E_{n}{ }^{8}\right\|\right)\right]$.

From Equation (11) and Equation (12), we obtain,

$$
\begin{aligned}
& \frac{F\left(x_{n}\right)}{F \prime\left(x_{n}\right)}=E_{n}-V_{2} E_{n}{ }^{2}+2\left(V_{2}^{2}-V_{3}\right) E_{n}{ }^{3}+\left(7 V_{2} V_{3}-\right. \\
& \left.3 V_{4}-4 V_{2}^{3}\right) E_{n}{ }^{4}+O\left(\left\|E_{n}{ }^{5}\right\|\right) .
\end{aligned}
$$

Substituting Equation (13) to Equation (9), it can be simplified as follows, since $E_{n}=x_{n}-x^{*}$,

$$
\begin{aligned}
y_{n}= & x^{*}+V_{2} E_{n}{ }^{2}+2\left(V_{3}-V_{2}^{2}\right) E_{n}{ }^{3}+\left(7 V_{2} V_{3}-3 V_{4}-\right. \\
& \left.4 V_{2}^{3}\right) E_{n}{ }^{4}+O\left(\left\|E_{n}{ }^{5}\right\|\right) .
\end{aligned}
$$

Step 2:

$$
\begin{aligned}
Z_{n}=y_{n}-1440\left[611 F^{\prime}\left(\frac{3 x_{n}+4 y_{n}}{7}\right)-\right. \\
453 F^{\prime}\left(\frac{-2 x_{n}+9 y_{n}}{7}\right)+ \\
562 F^{\prime}\left(\frac{-x_{n}+8 y_{n}}{7}\right)+562 F^{\prime}\left(\frac{x_{n}+6 y_{n}}{7}\right)- \\
453 F^{\prime}\left(\frac{2 x_{n}+5 y_{n}}{7}\right)+ \\
\left.611 F^{\prime}\left(\frac{-3 x_{n}+10 y_{n}}{7}\right)\right]^{-1} F\left(y_{n}\right) .
\end{aligned}
$$

By using the Taylor series, is can be obtained

$$
\begin{aligned}
F\left(y_{n}\right)= & F^{\prime}\left(x^{*}\right)\left[\left(y_{n}-x^{*}\right)+V_{2}\left(y_{n}-\right.\right. \\
& \left.x^{*}\right)^{2}+V_{3}\left(y_{n}-x^{*}\right)^{3}+ \\
& \left.O\left(\left\|E_{n}^{4}\right\|\right)\right] .
\end{aligned}
$$

Next, by substituting Equation (14) to Equation (16), we have,

$$
\begin{aligned}
F\left(y_{n}\right)= & F^{\prime}\left(x^{*}\right)\left[V_{2} E_{n}{ }^{2}+2\left(V_{3}-V_{2}^{2}\right) E_{n}{ }^{3}+\right. \\
& \left(7 V_{2} V_{3}-3 V_{4}-4 V_{2}^{3}\right) E_{n}{ }^{4}+ \\
& \left.V_{2}^{3} E_{n}{ }^{4}+O\left(\left\|E_{n}{ }^{5}\right\|\right)\right] .
\end{aligned}
$$

By using the Taylor series, we obtain:

$$
\begin{aligned}
F\left(w_{r}\right)= & F^{\prime}\left(x^{*}\right)\left[\left(w_{r}-x^{*}\right)+V_{2}\left(w_{r}-\right.\right. \\
& \left.x^{*}\right)^{2}+V_{3}\left(w_{r}-x^{*}\right)^{3}+ \\
& \left.O\left(\left\|E_{n}^{4}\right\|\right)\right] .
\end{aligned}
$$

and the derivative of Equation (18) is:

$$
\begin{aligned}
F^{\prime}\left(w_{r}\right)= & F^{\prime}\left(x^{*}\right)\left[I+2 V_{2}\left(w_{r}-x^{*}\right)+\right. \\
& \left.3 V_{3}\left(w_{r}-x^{*}\right)^{2}\right]+O\left(\left\|E_{n}^{3}\right\|\right) .
\end{aligned}
$$

where $r=1,2,3,4,5$, and 6 , and $w_{1}=\frac{3 x_{i}+4 y_{i}}{7}, w_{2}=$ $\frac{-2 x_{i}+9 y_{i}}{7}, w_{3}=\frac{-x_{i}+8 y_{i}}{7}, w_{4}=\frac{x_{i}+6 y_{i}}{7} ., w_{5}=\frac{2 x_{i}+5 y_{i}}{7}$, and $w_{6}=\frac{-3 x_{i}+10 y_{i}}{7}$.

Defined $\mathrm{T}=V_{2} E_{n}{ }^{2}+2\left(V_{3}-V_{2}^{2}\right) E_{n}{ }^{3}+\left(7 V_{2} V_{3}-3 V_{4}-\right.$ $\left.4 V_{2}^{3}\right) E_{n}{ }^{4}+O\left(\left\|E_{n}{ }^{5}\right\|\right)$ in Equation (14), we obtain

$$
y_{n}=x^{*}+\mathrm{T} \text {. }
$$

Substitute Equation (20) to $w_{1}, w_{2}, w_{3}, w_{4}, w_{5}$, and $w_{6}$, it can be obtained,

Furthermore, the derivative of Equation (11) is 
$w_{1}=\frac{3 x_{i}+4\left(x^{*}+\mathrm{T}\right)}{7}, w_{2}=\frac{-2 x_{i}+9\left(x^{*}+\mathrm{T}\right)}{7}, w_{3}=\frac{-x_{i}+8\left(x^{*}+\mathrm{T}\right)}{7}$,
$w_{4}=\frac{x_{i}+6\left(x^{*}+\mathrm{T}\right)}{7}, \quad w_{5}=\frac{2 x_{i}+5\left(x^{*}+\mathrm{T}\right)}{7}, \quad$ and $w_{6}=$
$\frac{-3 x_{i}+10\left(x^{*}+\mathrm{T}\right)}{7}$.

Next, the new, $w_{1}, w_{2}, w_{3}, w_{4}, w_{5}$, and $w_{6}$ are substituted to Equation (19). Supposed that $P_{n m}$ is a coefficient. We will obtain,

$$
\begin{aligned}
& F^{\prime}\left(w_{1}\right)=F^{\prime}\left(x^{*}\right)\left[I+\frac{6}{7} V_{2} E_{n}+P_{11} E_{n}{ }^{2}+P_{12} E_{n}{ }^{3}+\right. \\
& \left.P_{13} E_{n}{ }^{4}+O\left(\left\|E_{n}{ }^{5}\right\|\right)\right] \\
& F^{\prime}\left(w_{2}\right)=F^{\prime}\left(x^{*}\right)\left[I-\frac{4}{7} V_{2} E_{n}+P_{21} E_{n}{ }^{2}+P_{22} E_{n}{ }^{3}+P_{23} E_{n}{ }^{4}+\right. \\
& \left.O\left(\left\|E_{n}{ }^{5}\right\|\right)\right] \\
& F^{\prime}\left(w_{3}\right)=F^{\prime}\left(x^{*}\right)\left[I-\frac{2}{7} V_{2} E_{n}+P_{31} E_{n}{ }^{2}+P_{32} E_{n}{ }^{3}+P_{33} E_{n}{ }^{4}+\right. \\
& \left.O\left(\left\|E_{n}{ }^{5}\right\|\right)\right] \\
& F^{\prime}\left(w_{4}\right)=F^{\prime}\left(x^{*}\right)\left[I+\frac{2}{7} V_{2} E_{n}+P_{41} E_{n}{ }^{2}+P_{42} E_{n}{ }^{3}+P_{43} E_{n}{ }^{4}+\right. \\
& \left.O\left(\left\|E_{n}{ }^{5}\right\|\right)\right] \\
& F^{\prime}\left(w_{5}\right)=F^{\prime}\left(x^{*}\right)\left[I+\frac{4}{7} V_{2} E_{n}+P_{51} E_{n}{ }^{2}+P_{52} E_{n}{ }^{3}+\right. \\
& \left.P_{53} E_{n}{ }^{4}+O\left(\left\|E_{n}{ }^{5}\right\|\right)\right] . \\
& F^{\prime}\left(w_{6}\right)=F^{\prime}\left(x^{*}\right)\left[I-\frac{6}{7} V_{2} E_{n}+P_{61} E_{n}{ }^{2}+P_{62} E_{n}{ }^{3}+\right. \\
& \left.P_{63} E_{n}{ }^{4}+O\left(\left\|E_{n}{ }^{5}\right\|\right)\right] .
\end{aligned}
$$

Then,

$$
\begin{aligned}
& 611 F^{\prime}\left(w_{1}\right)-453 F^{\prime}\left(w_{2}\right)+562 F^{\prime}\left(w_{3}\right)+ \\
& 562 F^{\prime}\left(w_{4}\right)-453 F^{\prime}\left(w_{5}\right)+611 F^{\prime}\left(w_{6}\right)= \\
& 1440 F^{\prime}\left(x^{*}\right)\left[I+P_{1} E_{n}{ }^{2}+P_{2} E_{n}{ }^{3}+P_{3} E_{n}{ }^{4}+\right. \\
& \left.O\left(\left\|E_{n}{ }^{5}\right\|\right)\right] .
\end{aligned}
$$

We assume,

$611 P_{11}-453 P_{21}+562 P_{31}+562 P_{41}-453 P_{51}+611 P_{61}=P_{1}$ is a coefficient of $E_{n}{ }^{2}$

$611 P_{12}-453 P_{22}+562 P_{32}+562 P_{42}-453 P_{52}+611 P_{62}=P_{2}$ is a coefficient of $E_{n}{ }^{3}$

$611 P_{13}-453 P_{23}+562 P_{33}+562 P_{43}-453 P_{53}+611 P_{63}=P_{3}$ is a coefficient of $E_{n}{ }^{4}$

Substitute Equation (14) and Equation (21) to Equation (15), it is obtained

$$
\begin{gathered}
Z_{n}=x^{*}+P_{101} E_{n}{ }^{4}+P_{102} E_{n}{ }^{5}+P_{103} E_{n}{ }^{6}+ \\
P_{104} E_{n}{ }^{7}+P_{105} E_{n}{ }^{8}+O\left(\left\|E_{n}{ }^{9}\right\|\right) .
\end{gathered}
$$

Suppose the coefficient of $E_{n}{ }^{4}$ is $P_{101}, E_{n}{ }^{5}$ is $P_{102}, E_{n}{ }^{6}$ is $P_{103}, E_{n}{ }^{7}$ is $P_{104}$, and $E_{n}{ }^{8}$ is $P_{105}$

\section{Step 3:}

$$
x_{n+1}=Z_{n}-\frac{F\left(Z_{n}\right)}{F^{\prime}\left(Z_{n}\right)}
$$

By using the Taylor series, it is obtained

$$
\begin{gathered}
F\left(Z_{n}\right)=F^{\prime}\left(x^{*}\right)\left[\left(Z_{n}-x^{*}\right)+V_{2}\left(Z_{n}-x^{*}\right)^{2}+\right. \\
\left.V_{3}\left(Z_{n}-x^{*}\right)^{3}+O\left(\left\|E_{n}^{4}\right\|\right)\right] .
\end{gathered}
$$

Equation (22) is substituted to Equation (24)

$$
\begin{aligned}
F\left(Z_{n}\right)= & F^{\prime}\left(x^{*}\right)\left[P_{101} E_{n}{ }^{4}+P_{102} E_{n}{ }^{5}+\right. \\
& P_{103} E_{n}{ }^{6}+P_{104} E_{n}{ }^{7}+P_{105} E_{n}{ }^{8}+ \\
& \left.V_{2} P_{101}^{2} E_{n}{ }^{8}+O\left(\left\|E_{n}{ }^{9}\right\|\right)\right] .
\end{aligned}
$$

The derivatives Equation (24) is,

$$
\begin{aligned}
& F^{\prime}\left(Z_{n}\right)=F^{\prime}\left(x^{*}\right)\left[I+2 V_{2}\left(Z_{n}-x^{*}\right)+\right. \\
& \left.3 V_{3}\left(Z_{n}-x^{*}\right)^{2}+O\left(\left\|E_{n}{ }^{3}\right\|\right)\right] .
\end{aligned}
$$

Equation (22) is substituted to Equation (26). We have

$$
\begin{aligned}
F^{\prime}\left(Z_{n}\right)= & F^{\prime}\left(x^{*}\right)\left[I+2 V_{2}\left(P_{101} E_{n}{ }^{4}+\right.\right. \\
& P_{102} E_{n}{ }^{5}+P_{103} E_{n}{ }^{6}+P_{104} E_{n}{ }^{7}+ \\
& \left.O\left(\left\|E_{n}{ }^{8}\right\|\right)\right] .
\end{aligned}
$$

From Equation (25) and Equation (27). It is obtained

$$
\begin{gathered}
\frac{F\left(Z_{n}\right)}{F^{\prime}\left(Z_{n}\right)}=P_{101} E_{n}{ }^{4}+P_{102} E_{n}{ }^{5}+P_{103} E_{n}{ }^{6}+ \\
P_{104} E_{n}{ }^{7}+P_{105} E_{n}{ }^{8}-V_{2} P_{101}^{2} E_{n}{ }^{8}+ \\
O\left(\left\|E_{n}{ }^{9}\right\|\right) .
\end{gathered}
$$

Substitute Equation (22) and Equation (28) to Equation (23)

$$
\begin{aligned}
x_{n+1}= & x^{*}+P_{101} E_{n}{ }^{4}+P_{102} E_{n}{ }^{5}+P_{103} E_{n}{ }^{6}+ \\
& P_{104} E_{n}{ }^{7}+P_{105} E_{n}{ }^{8}+O\left(\left\|E_{n}{ }^{9}\right\|\right)-\left(P_{101} E_{n}{ }^{4}+\right. \\
& P_{102} E_{n}{ }^{5}+P_{103} E_{n}{ }^{6}+P_{104} E_{n}{ }^{7}+P_{105} E_{n}{ }^{8}- \\
& \left.V_{2} P_{101}^{2} E_{n}{ }^{8}+O\left(\left\|E_{n}{ }^{9}\right\|\right)\right) \\
x_{n+1}= & x^{*}+V_{2} P_{101}^{2} E_{n}{ }^{8}+O\left(\left\|E_{n}{ }^{9}\right\|\right) \\
\text { If } x_{n+1}- & x^{*}=E_{n+1}, \text { finally we will obtain, } \\
E_{n+1}= & O\left(\left\|E_{n}{ }^{8}\right\|\right),
\end{aligned}
$$

which shows that Algorithm \#1 (Equation (5)) has order of convergence 8 , the required results.

\section{Algorithm \#2}

Theorem 2: Let $x^{*}$ be a simple solution of the differentiable function $F(x)$ and given the initial value $x_{i}$, the three-steps scheme defined in Equation (6) has order of convergence 8 .

\section{Proof:}

Step 1: It is the same as step 1 of Algorithm \#1 (Equation (14)).

\section{Step 2:}

It has similar behavior as Algorithm \#1. We can find the Taylor series of $F\left(y_{n}\right)$ and $F^{\prime}\left(w_{r}\right)$ in step 2 of Equation (6), wherewhere $r=1,2,3,4,5,6$ and 7, and $w_{1}=$ $\frac{16 x_{n}-8 y_{n}}{8}, w_{2}=\frac{-3 x_{n}+11 y_{n}}{8}, w_{3}=\frac{25 x_{n}-17 y_{n}}{8}, w_{4}=$ $\frac{23 x_{n}-15 y_{n}}{8}, w_{5}=\frac{-2 x_{n}+10 y_{n}}{8}, w_{6}=\frac{3 x_{n}+5 y_{n}}{8}$, and $w_{7}=$ $\frac{-x_{n}+9 y_{n}}{8}$. 
Then substitute Equation (20) to $w_{1}, w_{2}, w_{3}, w_{4}, w_{5}, w_{6}$, and $w_{7}$, it is obtained,

$w_{1}=\frac{16 x_{n}-8\left(x^{*}+\mathrm{T}\right)}{8} \quad, \quad w_{2}=\frac{-3 x_{n}+11\left(x^{*}+\mathrm{T}\right)}{8} \quad, \quad w_{3}=$ $\frac{25 x_{n}-17\left(x^{*}+\mathrm{T}\right)}{8}, w_{4}=\frac{23 x_{n}-15\left(x^{*}+\mathrm{T}\right)}{8}, w_{5}=\frac{-2 x_{n}+10\left(x^{*}+\mathrm{T}\right)}{8}$, $w_{6}=\frac{3 x_{n}+5\left(x^{*}+\mathrm{T}\right)}{8}$ and $w_{7}=\frac{-x_{n}+9\left(x^{*}+\mathrm{T}\right)}{8}$.

Further, the new $w_{1}, w_{2}, w_{3}, w_{4}, w_{5}, w_{6}$, and $w_{7}$ are used to expand the Taylor series of $F^{\prime}\left(w_{r}\right)$. Supposed that $Q_{n m}$ is a coefficient. We will obtain,

$$
\begin{aligned}
& F^{\prime}\left(w_{1}\right)=F^{\prime}\left(x^{*}\right)\left[I+4 V_{2} E_{n}+Q_{11} E_{n}{ }^{2}+Q_{12} E_{n}{ }^{3}+\right. \\
& \left.Q_{13} E_{n}{ }^{4}+O\left(\left\|E_{n}{ }^{5}\right\|\right)\right] \\
& F^{\prime}\left(w_{2}\right)=F^{\prime}\left(x^{*}\right)\left[I-\frac{3}{4} V_{2} E_{n}+Q_{21} E_{n}{ }^{2}+Q_{22} E_{n}{ }^{3}+Q_{23} E_{n}{ }^{4}\right. \\
& \left.+O\left(\left\|E_{n}{ }^{5}\right\|\right)\right] \\
& F^{\prime}\left(w_{3}\right)=F^{\prime}\left(x^{*}\right)\left[I+\frac{25}{4} V_{2} E_{n}+Q_{31} E_{n}{ }^{2}+Q_{32} E_{n}{ }^{3}\right. \\
& +Q_{33} E_{n}{ }^{4}+O\left(\left\|E_{n}{ }^{5}\right\|\right) \\
& F^{\prime}\left(w_{4}\right)=F^{\prime}\left(x^{*}\right)\left[I+\frac{23}{4} V_{2} E_{n}+Q_{41} E_{n}{ }^{2}+Q_{42} E_{n}{ }^{3}+Q_{43} E_{n}{ }^{4}\right. \\
& \left.+O\left(\left\|E_{n}{ }^{5}\right\|\right)\right]
\end{aligned}
$$

Then,

$$
\begin{aligned}
& 460 F^{\prime}\left(w_{1}\right)-954 F^{\prime}\left(w_{2}\right)+2196 F^{\prime}\left(w_{3}\right)- \\
& 2496 F^{\prime}\left(w_{4}\right)+2196 F^{\prime}\left(w_{5}\right)- \\
& 954 F^{\prime}\left(w_{6}\right)+460 F^{\prime}\left(w_{7}\right)=945 F^{\prime}\left(x^{*}\right)[I+ \\
& \left.Q_{1} E_{n}{ }^{2}+Q_{2} E_{n}{ }^{3}+Q_{3} E_{n}{ }^{4}+O\left(\left\|E_{n}{ }^{5}\right\|\right)\right] .
\end{aligned}
$$

We assume,

$460 Q_{11}-954 Q_{21}+2196 Q_{31}-2496 Q_{41}+2196 Q_{51}-$ $954 Q_{61}+460 Q_{71}=Q_{1}$ is a coefficient of $E_{n}{ }^{2}$

$460 Q_{12}-954 Q_{22}+2196 Q_{32}-2496 Q_{42}+2196 Q_{52}-$ $954 Q_{62}+460 Q_{72}=Q_{2}$ is a coefficient of $E_{n}{ }^{3}$

$460 Q_{13}-954 Q_{23}+2196 Q_{33}-2496 Q_{43}+2196 Q_{53}-$ $954 Q_{63}+460 Q_{73}=Q_{3}$ is a coefficient of $E_{n}{ }^{4}$

Substitute Equation (14) and Equation (30) to step 2 of Equation (6), it is obtained

$$
\begin{aligned}
Z_{n}= & x^{*}+Q_{101} E_{n}{ }^{4}+Q_{102} E_{n}{ }^{5}+Q_{103} E_{n}{ }^{6}+ \\
& Q_{104} E_{n}{ }^{7}+Q_{105} E_{n}{ }^{8}+O\left(\left\|E_{n}{ }^{9}\right\|\right) .
\end{aligned}
$$

Suppose the coefficient of $E_{n}{ }^{4}$ is $Q_{101}, E_{n}{ }^{5}$ is $Q_{102}, E_{n}{ }^{6}$ is $Q_{103}, E_{n}{ }^{7}$ is $Q_{104}$, and $E_{n}{ }^{8}$ is $Q_{105}$.
Step 3: We can easily find the Taylor series of $F\left(Z_{n}\right)$ and $F^{\prime}\left(Z_{n}\right)$ of step 3 in Equation (6) since $Z_{n}$ has been defined in Equation (31). Furthermore, we can also obtain

$$
\begin{gathered}
\frac{F\left(Z_{n}\right)}{F^{\prime}\left(Z_{n}\right)}=Q_{101} E_{n}{ }^{4}+Q_{102} E_{n}{ }^{5}+Q_{103} E_{n}{ }^{6}+ \\
Q_{104} E_{n}{ }^{7}+Q_{105} E_{n}{ }^{8}- \\
V_{2} Q_{101}^{2} E_{n}{ }^{8}+O\left(\left\|E_{n}{ }^{9}\right\|\right) .
\end{gathered}
$$

Finally, Equation (31) and Equation (32) are substituted to step 3 of Equation (6) we obtain,

$$
\begin{aligned}
& x_{n+1}= x^{*}+Q_{101} E_{n}{ }^{4}+Q_{102} E_{n}{ }^{5}+Q_{103} E_{n}{ }^{6}+ \\
& Q_{104} E_{n}{ }^{7}+Q_{105} E_{n}{ }^{8}+O\left(\left\|E_{n}{ }^{9}\right\|\right)- \\
&\left(Q_{101} E_{n}{ }^{4}+Q_{102} E_{n}{ }^{5}+Q_{103} E_{n}{ }^{6}+Q_{104} E_{n}{ }^{7}+\right. \\
&\left.Q_{105} E_{n}{ }^{8}-V_{2} Q_{101}^{2} E_{n}{ }^{8}+O\left(\left\|E_{n}{ }^{9}\right\|\right)\right) \\
& x_{n+1}= x^{*}+V_{2} Q_{101}^{2} E_{n}{ }^{8}+O\left(\left\|E_{n}{ }^{9}\right\|\right) \\
& \text { If } x_{n+1}-x^{*}=E_{n+1}, \text { Finally we have } \\
& E_{n+1}=O\left(\left\|E_{n}{ }^{8}\right\|\right) .
\end{aligned}
$$

which shows that Algorithm \#2 (Equation (6)) has order of convergence 8 , the required results.

\section{Algorithm \#3}

Theorem 3: Let $x^{*}$ be a simple solution of the differentiable function $F(x)$ and given the initial value $x_{i}$, the three-steps scheme defined in Equation (7) has order of convergence 8 .

\section{Proof:}

Step 1: It is the same as step 1 of Algorithm \#1 (Equation (14)).

Step 2: We can find the Taylor series of $F\left(y_{n}\right)$ and $F^{\prime}\left(w_{r}\right)$ in step 2 of Equation (7), where $r=1,2,3$, and 4 and $w_{1}=\frac{7 x_{n}-y_{n}}{6}, w_{2}=\frac{2 x_{n}+4 y_{n}}{6}, w_{3}=\frac{-2 x_{n}+8 y_{n}}{6}$, and $w_{4}=\frac{-7 x_{n}+13 y_{n}}{6}$.

Then substitute Equation (20) to $w_{1}, w_{2}, w_{3}$, and $w_{4}$, it is obtained

$w_{1}=\frac{7 x_{n}-\left(x^{*}+\mathrm{T}\right)}{6}, w_{2}=\frac{2 x_{n}+4\left(x^{*}+\mathrm{T}\right)}{6}, w_{3}=\frac{-2 x_{n}+8\left(x^{*}+\mathrm{T}\right)}{6}$, and $w_{4}=\frac{-7 x_{n}+13\left(x^{*}+\mathrm{T}\right)}{6}$.

The new $w_{1}, w_{2}, w_{3}$, and $w_{4}$ are used to expand the Taylor series of $F^{\prime}\left(w_{r}\right)$. Supposed that $L_{n m}$ is a coefficient. We will obtain,

$$
\begin{aligned}
& F^{\prime}\left(w_{1}\right)=F^{\prime}\left(x^{*}\right)\left[I+\frac{7}{3} V_{2} E_{n}+L_{11} E_{n}{ }^{2}+L_{12} E_{n}{ }^{3}+\right. \\
& \left.L_{13} E_{n}{ }^{4}+O\left(\left\|E_{n}{ }^{5}\right\|\right)\right] \\
& F^{\prime}\left(w_{2}\right)=F^{\prime}\left(x^{*}\right)\left[I+\frac{2}{3} V_{2} E_{n}+L_{21} E_{n}{ }^{2}+L_{22} E_{n}{ }^{3}+\right. \\
& \left.L_{23} E_{n}{ }^{4}+O\left(\left\|E_{n}{ }^{5}\right\|\right)\right] \\
& F^{\prime}\left(w_{3}\right)=F^{\prime}\left(x^{*}\right)\left[I-\frac{2}{3} V_{2} E_{n}+L_{31} E_{n}{ }^{2}+L_{32} E_{n}{ }^{3}+L_{33} E_{n}{ }^{4}+\right. \\
& \left.O\left(\left\|E_{n}{ }^{5}\right\|\right)\right]
\end{aligned}
$$


$F^{\prime}\left(w_{4}\right)=F^{\prime}\left(x^{*}\right)\left[I-\frac{7}{3} V_{2} E_{n}+L_{41} E_{n}{ }^{2}+L_{42} E_{n}{ }^{3}+\right.$ $\left.L_{43} E_{n}{ }^{4}+O\left(\left\|E_{n}{ }^{5}\right\|\right)\right]$.

Then,

$$
\begin{aligned}
& 11 F^{\prime}\left(w_{1}\right)+F^{\prime}\left(w_{2}\right)+F^{\prime}\left(w_{3}\right)+ \\
& 11 F^{\prime}\left(w_{4}\right)=24 F^{\prime}\left(x^{*}\right)\left[I+L_{1} E_{n}{ }^{2}+\right. \\
& \left.L_{2} E_{n}{ }^{3}+L_{3} E_{n}{ }^{4}+O\left(\left\|E_{n}{ }^{5}\right\|\right)\right] .
\end{aligned}
$$

It is assumed,

$11 L_{11}+L_{21}+L_{31}+11 L_{41}=L_{1}$ is a coefficient of $E_{n}{ }^{2}$

$11 L_{12}+L_{22}+L_{32}+11 L_{42}=L_{2}$ is a coefficient of $E_{n}{ }^{3}$

$11 L_{13}+L_{23}+L_{33}+11 L_{43}=L_{3}$ is a coefficient oft $E_{n}{ }^{4}$

Substitute Equation (14) and Equation (33) to step 2 of Equation (7), it is obtained

$$
\begin{gathered}
Z_{n}=x^{*}+L_{101} E_{n}{ }^{4}+L_{102} E_{n}{ }^{5}+L_{103} E_{n}{ }^{6}+ \\
L_{104} E_{n}{ }^{7}+L_{105} E_{n}{ }^{8}+O\left(\left\|E_{n}{ }^{9}\right\|\right) .
\end{gathered}
$$

Suppose the coefficient of $E_{n}{ }^{4}$ is $L_{101}, E_{n}{ }^{5}$ is $L_{102}, E_{n}{ }^{6}$ is $L_{103}, E_{n}{ }^{7}$ is $L_{104}$, and $E_{n}{ }^{8}$ is $L_{105}$

Step 3: We can easily find the Taylor series of $F\left(Z_{n}\right)$ and $F^{\prime}\left(Z_{n}\right)$ of step 3 in Equation (7) since $Z_{n}$ has been defined in Equation (34). Furthermore, we can also obtainWe can easily find the Taylor series of $F\left(Z_{n}\right)$ and $F^{\prime}\left(Z_{n}\right)$ of step 3 in Equation (6) since $Z_{n}$ has been defined by Equation (31). Furthermore, we can also obtain

$$
\begin{aligned}
\frac{F\left(Z_{n}\right)}{F^{\prime}\left(Z_{n}\right)}= & L_{101} E_{n}{ }^{4}+L_{102} E_{n}{ }^{5}+L_{103} E_{n}{ }^{6}+ \\
& L_{104} E_{n}{ }^{7}+L_{105} E_{n}{ }^{8}-V_{2} L_{101}{ }^{2} E_{n}{ }^{8}+ \\
& O\left(\left\|E_{n}{ }^{9}\right\|\right) .
\end{aligned}
$$

Finally, Equation (34) and Equation (35) are substituted to step 3 of Equation (7) we obtain,

$$
\begin{aligned}
x_{n+1}= & x^{*}+L_{101} E_{n}{ }^{4}+L_{102} E_{n}{ }^{5}+L_{103} E_{n}{ }^{6}+ \\
& L_{104} E_{n}{ }^{7}+L_{105} E_{n}{ }^{8}+O\left(\left\|E_{n}{ }^{9}\right\|\right)-\left(L_{101} E_{n}{ }^{4}+\right. \\
& L_{102} E_{n}{ }^{5}+L_{103} E_{n}{ }^{6}+L_{104} E_{n}{ }^{7}+L_{105} E_{n}{ }^{8}- \\
& \left.V_{2} L_{101}^{2} E_{n}{ }^{8}+O\left(\left\|E_{n}{ }^{9}\right\|\right)\right) \\
x_{n+1}= & x^{*}+V_{2} L_{101}^{2} E_{n}{ }^{8}+O\left(\left\|E_{n}{ }^{9}\right\|\right)
\end{aligned}
$$

If $x_{n+1}-x^{*}=E_{n+1}$ then we have $E_{n+1}=O\left(\left\|E_{n}{ }^{8}\right\|\right)$.

which shows that Algorithm \#3 (Equation (7)) has order of convergence 8 , the required results.

\section{Algorithm \#4}

Theorem 4: Let $x^{*}$ be a simple solution of the differentiable function $F(x)$ and given the initial value $x_{i}$, the three-steps scheme defined in Equation (8) has order of convergence 8 .

\section{Proof:}

Step 1: It is the same as step 1 of Algorithm \#1 (Equation (14)).
Step 2: We can find the Taylor series of $F\left(y_{n}\right)$ and $F^{\prime}\left(w_{r}\right)$ in step 2 of Equation (8), where $r=1,2,3,4$, and 5 , and $w_{1}=\frac{28 x_{n}-20 y_{n}}{8}, w_{2}=\frac{11 x_{n}-3 y_{n}}{8}, w_{3}=\frac{-5 x_{n}+13 y_{n}}{8}$, $w_{4}=\frac{19 x_{n}-11 y_{n}}{8}$, dan $w_{5}=\frac{22 x_{n}-14 y_{n}}{8}$.

Then substitute Equation (20) to $w_{1}, w_{2}, w_{3}, w_{4}$ and $w_{5}$, it is obtained

$w_{1}=\frac{28 x_{n}-20\left(x^{*}+\mathrm{T}\right)}{8} \quad, \quad w_{2}=\frac{11 x_{n}-3\left(x^{*}+\mathrm{T}\right)}{8}, \quad, \quad w_{3}=$
$\frac{-5 x_{n}+13\left(x^{*}+\mathrm{T}\right)}{8}, \quad w_{4}=\frac{19 x_{n}-11\left(x^{*}+\mathrm{T}\right)}{8}, \quad$ and $\quad w_{5}=$
$\frac{22 x_{n}-14\left(x^{*}+\mathrm{T}\right)}{8}$

Now, the new $w_{1}, w_{2}, w_{3}, w_{4}$ and $w_{5}$ are used to expand the Taylor series of $F^{\prime}\left(w_{r}\right)$. Supposed that $M_{n m}$ is a coefficient. We will obtain,

$$
\begin{aligned}
& F^{\prime}\left(w_{1}\right)=F^{\prime}\left(x^{*}\right)\left[I+7 V_{2} E_{n}+M_{11} E_{n}{ }^{2}+M_{12} E_{n}{ }^{3}+\right. \\
& \left.M_{13} E_{n}{ }^{4}+O\left(\left\|E_{n}{ }^{5}\right\|\right)\right] \\
& F^{\prime}\left(w_{2}\right)=F^{\prime}\left(x^{*}\right)\left[I+\frac{11}{4} V_{2} E_{n}+M_{21} E_{n}{ }^{2}+M_{22} E_{n}{ }^{3}+\right. \\
& \left.M_{23} E_{n}{ }^{4}+O\left(\left\|E_{n}{ }^{5}\right\|\right)\right] \\
& F^{\prime}\left(w_{3}\right)=F^{\prime}\left(x^{*}\right)\left[I-\frac{5}{4} V_{2} E_{n}+M_{31} E_{n}{ }^{2}+M_{32} E_{n}{ }^{3}+\right. \\
& \left.M_{33} E_{n}{ }^{4}+O\left(\left\|E_{n}{ }^{5}\right\|\right)\right] \\
& F^{\prime}\left(w_{4}\right)=F^{\prime}\left(x^{*}\right)\left[I+\frac{19}{4} V_{2} E_{n}+M_{41} E_{n}{ }^{2}+M_{42} E_{n}{ }^{3}+\right. \\
& \left.M_{43} E_{n}{ }^{4}+O\left(\left\|E_{n}{ }^{5}\right\|\right)\right]
\end{aligned}
$$

$F^{\prime}\left(w_{5}\right)=F^{\prime}\left(x^{*}\right)\left[I+\frac{11}{2} V_{2} E_{n}+M_{51} E_{n}{ }^{2}+M_{52} E_{n}{ }^{3}+\right.$ $\left.M_{53} E_{n}{ }^{4}+O\left(\left\|E_{n}{ }^{5}\right\|\right)\right]$

Then,

$$
\begin{aligned}
& 11 F^{\prime}\left(w_{1}\right)-14 F^{\prime}\left(w_{2}\right)+26 F^{\prime}\left(w_{3}\right)- \\
& 14 F^{\prime}\left(w_{4}\right)+11 F^{\prime}\left(w_{5}\right)=20 F^{\prime}\left(x^{*}\right)[I+ \\
& \left.M_{1} E_{n}{ }^{2}+M_{2} E_{n}{ }^{3}+M_{3} E_{n}{ }^{4}+O\left(\left\|E_{n}{ }^{5}\right\|\right)\right] .
\end{aligned}
$$

It is assumed,

$11 M_{11}-14 M_{21}+26 M_{31}-14 M_{41}+11 M_{51}=M_{1}$ is a coefficient of $E_{n}{ }^{2}$

$11 M_{12}-14 M_{22}+26 M_{32}-14 M_{42}+11 M_{52}=M_{2}$ is a coefficient of $E_{n}{ }^{3}$

$11 M_{13}-14 M_{23}+26 M_{33}-14 M_{43}+11 M_{53}=M_{3}$ is a coefficient of $E_{n}{ }^{4}$

Substitute Equation (14) and Equation (37) to step 2 of Equation (8), it is obtained

$$
\begin{aligned}
Z_{n}= & x^{*}+M_{101} E_{n}{ }^{4}+M_{102} E_{n}{ }^{5}+M_{103} E_{n}{ }^{6}+ \\
& M_{104} E_{n}{ }^{7}+M_{105} E_{n}{ }^{8}+O\left(\left\|E_{n}{ }^{9}\right\|\right) .
\end{aligned}
$$

Suppose the coefficient of $E_{n}{ }^{4}$ is $M_{101}, E_{n}{ }^{5}$ is $M_{102}, E_{n}{ }^{6}$ is $M_{103}, E_{n}{ }^{7}$ is $M_{104}$, and $E_{n}{ }^{8}$ is $M_{105}$.

Step 3: Since $Z_{n}$ has been defined in Equation (38), we can find the convergence of the algorithm using similar way as previous algorithm as follows 
$Z_{n} \quad x_{n+1}=x^{*}+M_{101} E_{n}{ }^{4}+M_{102} E_{n}{ }^{5}+M_{103} E_{n}{ }^{6}+$ $M_{104} E_{n}{ }^{7}+M_{105} E_{n}{ }^{8}+O\left(\left\|E_{n}{ }^{9}\right\|\right)-\left(M_{101} E_{n}{ }^{4}+\right.$

$M_{102} E_{n}{ }^{5}+M_{103} E_{n}{ }^{6}+M_{104} E_{n}{ }^{7}+M_{105} E_{n}{ }^{8}-$

$\left.V_{2} M_{101}^{2} E_{n}{ }^{8}+O\left(\left\|E_{n}{ }^{9}\right\|\right)\right)$

$x_{n+1}=x^{*}+V_{2} M_{101}^{2} E_{n}{ }^{8}+O\left(\left\|E_{n}{ }^{9}\right\|\right)$

If $x_{n+1}-x^{*}=E_{n+1}$, then, $E_{n+1}=O\left(\left\|E_{n}^{8}\right\|\right)$.

which shows that Algorithm \#3 (Equation (7)) has order of convergence 8 , the required results.

\subsection{Numerical Results}

In this study, three simple non-linear equation systems examples are given. The examples are solved with the other multistep methods, such as FSM, KHM, Farida's best algorithm [1], and the proposed algorithm. The convergence criteria or error tolerance $(\varepsilon)$ is $\varepsilon \leq$ $10^{-15}$ and the maximum iteration is 50 . The comparison results are shown in Tables 1 - 3 below.
Example 1. Initial value $(1,1,1)$

$$
\begin{aligned}
& 2 x^{2}+y-z^{2}-10=0 \\
& 3 x^{2}+6 y-z^{2}-2=0 \\
& x^{2}-5 y+6 z^{2}-4=0
\end{aligned}
$$

Example 2. initial value $(-1,1,-1)$

$$
\begin{aligned}
& 10 x+\sin (x+y)-1=0 \\
& 8 y-(\cos (z-y))^{2}-1=0 \\
& 12 z+\sin z-1=0
\end{aligned}
$$

\begin{tabular}{|c|c|c|c|}
\hline Method & Iter & Solution & The function value \\
\hline KHM1 & 5 & $\mathrm{x}=2.183031149571622, \mathrm{y}=2.046875000000000, \mathrm{z}=1.256234452640111$ & $\mathrm{f}_{-} 1=-0.00000000035 \times 10^{\wedge}(-5), \mathrm{f} \_2=-0.00000000071 \times 10^{\wedge}(-5), \mathrm{f} \_3=-0.00000000071 \times 10^{\wedge}(-5)$ \\
\hline FSM & 6 & $\mathrm{x}=2.183031149571622, \mathrm{y}=2.046875000000000, \mathrm{z}=1.256234452640111$ & f_1 $1=-0.00000000035 \times 10^{\wedge}(-5)$, f $\_2=-0.00000000071 \times 10^{\wedge}(-5)$, f $\_3=-0.00000000071 \times 10^{\wedge}(-5)$ \\
\hline $\begin{array}{l}\text { The best Farida's } \\
\text { method }\end{array}$ & 5 & $\mathrm{x}=2.183031149571622, \mathrm{y}=2.046875000000000, \mathrm{z}=1.256234452640111$ & $\mathrm{f}_{-} 1=-0.00000000035 \times 10^{\wedge}(-5), \mathrm{f} \_2=-0.00000000071 \times 10^{\wedge}(-5), \mathrm{f} \_3=-0.00000000071 \times 10^{\wedge}(-5)$ \\
\hline Algorithm \#1 & 3 & $\mathrm{x}=2.183031149571622, \mathrm{y}=2.046875000000000, \mathrm{z}=1.256234452640111$ & $\mathrm{f} \_1=-0.00000000035 \times 10^{\wedge}(-5), \mathrm{f} \_2=-0.00000000035 \times 10^{\wedge}(-5), \mathrm{f} \_3=-0.00000000035 \times 10^{\wedge}(-5)$ \\
\hline Algorithm \#2 & 3 & $\mathrm{x}=2.183031149571622, \mathrm{y}=2.046875000000000, \mathrm{z}=1.256234452640111$ & $\mathrm{f} \_1=-0.00000000035 \times 10^{\wedge}(-5), \mathrm{f} \_2=-0.00000000035 \times 10^{\wedge}(-5), \mathrm{f} \_3=-0.00000000035 \times 10^{\wedge}(-5)$ \\
\hline Algorithm \#3 & 3 & $\mathrm{x}=2.183031149571622, \mathrm{y}=2.046875000000000, \mathrm{z}=1.256234452640111$ & $\mathrm{f}_{-} 1=0.000000000355 \times 10^{\wedge}(-5), \mathrm{f}_{-} 2=0.000000000355 \times 10^{\wedge}(-5), f_{-} 3=-0.00000000035 \times 10^{\wedge}(-5)$ \\
\hline Algorithm \#4 & 3 & $\mathrm{x}=2.183031149571622, \mathrm{y}=2.046875000000000, \mathrm{z}=1.256234452640111$ & f_1 $1=-0.00000000035 \times 10^{\wedge}(-5)$, f $\_2=-0.00000000035 \times 10^{\wedge}(-5)$, f $\_3=-0.00000000035 \times 10^{\wedge}(-5)$ \\
\hline
\end{tabular}

\begin{tabular}{|c|c|c|c|}
\hline Method & Iter & Solution & The function value \\
\hline KHM1 & 4 & $\mathrm{x}=0.068978349172667, \mathrm{y}=0.246442418609183, \mathrm{z}=0.076928911987537$ & f_1 $1=0.000000000488 \times 10^{\wedge}(-5)$, f $\_2=0.000000000044 \times 10^{\wedge}(-5)$, f $\_3=0.000000000044 \times 10^{\wedge}(-5)$ \\
\hline FSM & 6 & $\mathrm{x}=0.068978349172667, \mathrm{y}=0.246442418609183, \mathrm{z}=0.076928911987537$ & f $\_1=0.000000000488 \times 10^{\wedge}(-5)$, f $\_2=0.000000000044 \times 10^{\wedge}(-5), f \_3=0.000000000044 \times 10^{\wedge}(-5)$ \\
\hline $\begin{array}{l}\text { The best Farida's } \\
\text { method }\end{array}$ & 4 & $x=0.068978349172667, y=0.246442418609183, z=0.076928911987537$ & f_1 $1=0.000000000488 \times 10^{\wedge}(-5)$, f $\_2=0.000000000044 \times 10^{\wedge}(-5)$, f $\_3=0.000000000044 \times 10^{\wedge}(-5)$ \\
\hline Algorithm \#1 & 3 & $\mathrm{x}=0.068978349172667, \mathrm{y}=0.246442418609183, \mathrm{z}=0.076928911987537$ & f $\_1=0.000000000488 \times 10^{\wedge}(-5)$, f $\_2=0.000000000044 \times 10^{\wedge}(-5), f \_3=0.000000000044 \times 10^{\wedge}(-5)$ \\
\hline Algorithm \#3 & 3 & $\mathrm{x}=0.068978349172667, \mathrm{y}=0.246442418609183, \mathrm{z}=0.076928911987537$ & f $\_1=0.000000000488 \times 10^{\wedge}(-5)$, f $\_2=0.000000000044 \times 10^{\wedge}(-5), f \_3=0.000000000044 \times 10^{\wedge}(-5)$ \\
\hline Algorithm \#4 & 3 & $\mathrm{x}=0.068978349172667, \mathrm{y}=0.246442418609183, \mathrm{z}=0.076928911987537$ & $f_{-} 1=0.000000000488 \times 10^{\wedge}(-5), f \_2=0.000000000044 \times 10^{\wedge}(-5), f_{-} 3=0.000000000044 \times 10^{\wedge}(-5)$ \\
\hline
\end{tabular}

Example 3. initial value $(0,0,0)$

$$
\begin{aligned}
& 15 x+y^{2}-4 z-13=0 \\
& x^{2}+10 y-e^{-z}-11=0 \\
& y^{3}-25 z+22=0
\end{aligned}
$$

\begin{tabular}{|c|c|c|c|}
\hline Method & Iter & Solution & The function value \\
\hline KHM1 & 4 & $\mathrm{x}=1.042149560576938, \mathrm{y}=1.031091271839402, \mathrm{z}=0.923848154879368$ & f $\_1=-0.0000000005 \times 10^{\wedge}(-5)$, f $\_2=-0.0000000001 \times 10^{\wedge}(-5)$, f $\_3=-0.0000000007 \times 10^{\wedge}(-5)$ \\
\hline FSM & 5 & $\mathrm{x}=1.042149560576938, \mathrm{y}=1.031091271839402, \mathrm{z}=0.923848154879368$ & f_1 $1=-0.0000000005 \times 10^{\wedge}(-5)$, f $\_2=-0.0000000001 \times 10^{\wedge}(-5)$, f $\_3=-0.0000000007 \times 10^{\wedge}(-5)$ \\
\hline $\begin{array}{l}\text { The best Farida's } \\
\text { method }\end{array}$ & 4 & $\mathrm{x}=1.042149560576938, \mathrm{y}=1.031091271839402, \mathrm{z}=0.923848154879368$ & f_1 $1=-0.0000000005 \times 10^{\wedge}(-5)$, f $\_2=-0.0000000001 \times 10^{\wedge}(-5)$, f $\_3=-0.0000000007 \times 10^{\wedge}(-5)$ \\
\hline Algorithm \#1 & 3 & $x=1.042149560576938, y=1.031091271839402, z=0.923848154879368$ & f $\_1=-0.0000000005 \times 10^{\wedge}(-5)$, f $\_2=-0.0000000001 \times 10^{\wedge}(-5)$, f $\_3=-0.0000000007 \times 10^{\wedge}(-5)$ \\
\hline Algorithm \#2 & 3 & $\mathrm{x}=1.042149560576938, \mathrm{y}=1.031091271839402, \mathrm{z}=0.923848154879368$ & f $\_1=-0.0000000005 \times 10^{\wedge}(-5)$, f $\_2=-0.0000000001 \times 10^{\wedge}(-5)$, f $\_3=-0.0000000007 \times 10^{\wedge}(-5)$ \\
\hline Algorithm \#3 & 3 & $x=1.042149560576938, y=1.031091271839402, z=0.923848154879368$ & $\mathrm{f} \_1=-0.0000000005 \times 10^{\wedge}(-5), \mathrm{f} \_2=-0.0000000001 \times 10^{\wedge}(-5), \mathrm{f} \_3=-0.0000000007 \times 10^{\wedge}(-5)$ \\
\hline Algorithm \#4 & 3 & $\mathrm{x}=1.042149560576938, \mathrm{y}=1.031091271839402, \mathrm{z}=0.923848154879368$ & $\mathrm{f} \_1=-0.0000000005 \times 10^{\wedge}(-5)$, f $\_2=-0.0000000001 \times 10^{\wedge}(-5)$, f $\_3=-0.0000000007 \times 10^{\wedge}(-5)$ \\
\hline
\end{tabular}

Table 1. Comparison results of Example 1

Table 2. Comparison results of Example 2

Table 3. Comparison results of Example 3 
Tables 1 - 3 show that the proposed algorithm converge faster than the references in use.

\section{CONCLUSION}

In this study, we propose four new three-steps NewtonRaphson-like algorithms. It has been proven that the proposed algorithms have the order of convergence 8 . Numerical examples show that the proposed algorithms converge faster than the other multistep algorithms, such as the FSM method, KHM method, and Farida's best method.

\section{REFERENCES}

[1] M.Z. Arif, Y. Farida, A. Kamsyakawuni, Penerapan Metode Newton-Cotes Open Form 5 Titik untuk Menyelesaikan Sistem Persamaan Nonlinier (in Indonesian), Unnes Journal of Mathematics, 2017, 6(1), pp. 102-107.

[2] M. Frontini, E. Sormani, Some variant of Newton's method with third-order convergence. Applied Mathematics and Computation, 2003, vol. 140, pp. 419-426.
[3] M.T. Darvishi, A. Barati, A Third-Order Newtontype Method to Solve Systems of Nonlinear Equations, Applied Mathematics and Computation, 2007, vol. 187, pp. 630-635.

[4] M.T. Darvishi, A. Barati, Super Cubic Iterative Methods to Solve Systems of Nonlinear Equations, Applied Mathematics and Computation, 2007, vol. 188, pp. 1678-1685.

[5] A. Cordero, J.L. Hueso, E. Martínez, J.R. Torregrosa, Increasing the Convergence Order of An Iterative Method for Nonlinear System, Applied Mathematics Letters, 2012, vol. 25, pp. 2369-2374.

[6] J.L. Hueso, E. Martínez, J.R. Torregrosa, Third Order Iterative Methods Free from Second Derivative for Nonlinear Systems, Applied Mathematics and Computation, 2009, vol. 215, pp. 58-65.

[7] M.Q. Khirallah, M.A. Hafiz, Novel Three Order Methods for Solving a System of Nonlinear Equations, Bulletin of Mathematical Sciences \& Applications, 2012, 1 (2), pp. 1-14. 